\title{
A radiation tolerant readout link and control board for the Phase-II upgrade of the ATLAS Hadronic Tile Calorimeter
}

Tigran Mkrtchyan, on behalf of the ATLAS Tile Calorimeter System

\begin{abstract}
We present performance and radiation test results for the updated prototype of a radiation tolerant read-out link and control board (Daughterboard) for the Phase-II upgrade of the ATLAS Hadronic Tile Calorimeter (TileCal) front-end electronics for high-luminosity LHC. In the upgraded system, photomultiplier signals are amplified, shaped and digitized by the front-end electronics and a Mainboard (MB), while the Daughterboard (DB) collects and transmits the digitized data off-detector over multigigabit optical links. The DB provides a bidirectional interface between the front-end and off-detector electronics, receiving configuration, control and LHC-synchronous timing over two 4.8 Gbps downlinks to a pair of CERN GBTx ASICs, and providing high-speed readout off-detector over two redundant 9.6 Gbps uplinks, each driven by one of two Xilinx Kintex Ultrascale FPGAs. The DB design is double redundant to minimise single-point failure modes, and the FPGA firmware uses strategies including TMR, FEC and CRC to minimize the effects of single-event upsets and damage from hadronic and minimum ionizing radiation. The DB is expected to receive approximately $0.2 \mathrm{krad}$ of total ionizing and $2.44 \times 10^{11}$ neutrons $/ \mathrm{cm}^{2}$ of non-ionising radiation over a 10 year period, so the prototype board and components must pass TID, NIEL and SEE with large safety factors to be radiation qualified for high-luminosity LHC operations.
\end{abstract}

\section{INTRODUCTION}

The High Luminosity Large Hadron Collider (HL-LHC) is planned to have an increase in the instantaneous luminosity by a factor of 5-7 which will increase the pile-up to as many as 200 proton collisions per bunch crossing. In preparation for the HL-LHC the ATLAS detector (Fig. 11a) will be upgraded to cope with the high radiation environment and data processing demands. The ATLAS Tile Calorimeter (Fig. 1 $\mathrm{b}$ ) is a hadronic sampling calorimeter made of alternating layers of steel absorber plates and plastic scintillator tiles. The whole calorimeter is composed of 4 cylindrical barrels (Fig. 16) with 64 wedge-shaped modules in each. The modules are segmented radially in 3 layers and in pseudo-projective cells. The light produced by the scintillating tiles is collected on both sides of each cell, and guided by wavelength shifting fibers to two photomultiplier tubes (PMTs) (Fig. 11).

The aging components of TileCal are incompatible with the new trigger architecture and will not be able to handle the increased rates of pile-up, thus arise the need to upgrade the readout and trigger system [1].

口

T. Mkrtchyan is with the Alikhanyan National Science Laboratory, Yerevan, Armenia (email: tigran.mkrtchyan@cern.ch)
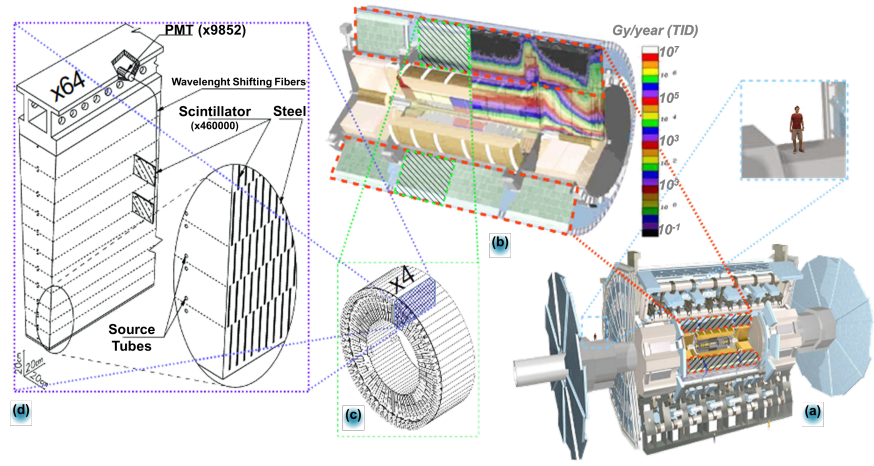

Fig. 1. The ATLAS Tile Calorimeter.

\section{TileCal's Phase-II ReAd-OUt Electronics}

The TileCal Phase-II upgrade will fully replace the current readout electronics to provide fully digital data to the offdetector systems at $40 \mathrm{MHz}$. To minimize single point failures each TileCal wedge-shaped module will house 4 independently operating Minidrawers (MDs). Each MD (Fig. 2) contains up to 12 PMT blocks, one Mainboard (MB) and one Daughterboard (DB). The PMT signals are shaped and amplified by Front-End boards (FEBs), before being digitized by a Mainboard in two gains with 12-bit at 40 Msps. The DB continuously reads out and formats the data from the MB before sending them to the off-detector systems. In addition, the DB receives and distributes the LHC clocks, configuration and control commands to the MB, all through optical multiGbps links.

On the off-detector
side, the TileCal Preprocessors receive, reconstruct and store the digital data in the pipelines while waiting for the trigger decision, as well as distribute the LHC clock to the DBs.

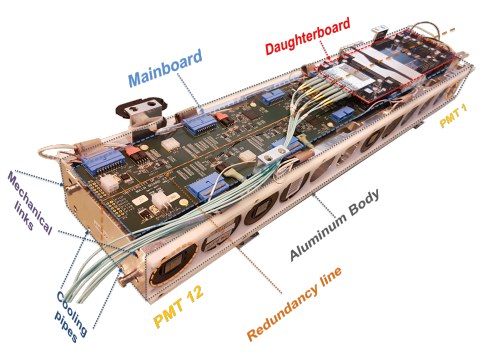

Fig. 2. TileCal Minidrawer. 


\section{READOUT LINK AND CONTROL BOARD \\ (DAUGHTERBOARD)}

The DB revision 5 (DB5) (Fig. 3) design is divided into two independent sides to provide redundancy and minimize of single point failures. Each side is powered by a GTY equipped Kintex Ultrascale+ FPGA that controls all the board interfaces, and a GBTx [2] ASIC chip to provide good quality LHC syncronized clocks, remote JTAG access, as well as configuration and control buses. The DB5 contains XADC interfaces for monitoring external sensors, and unique serial ID 48-bit chips for identification. The DB5 design also retains legacy interfaces for the High Voltage, Mainboard FPGA Mezzanine Card (FMC) interface and the Cs calibration System.

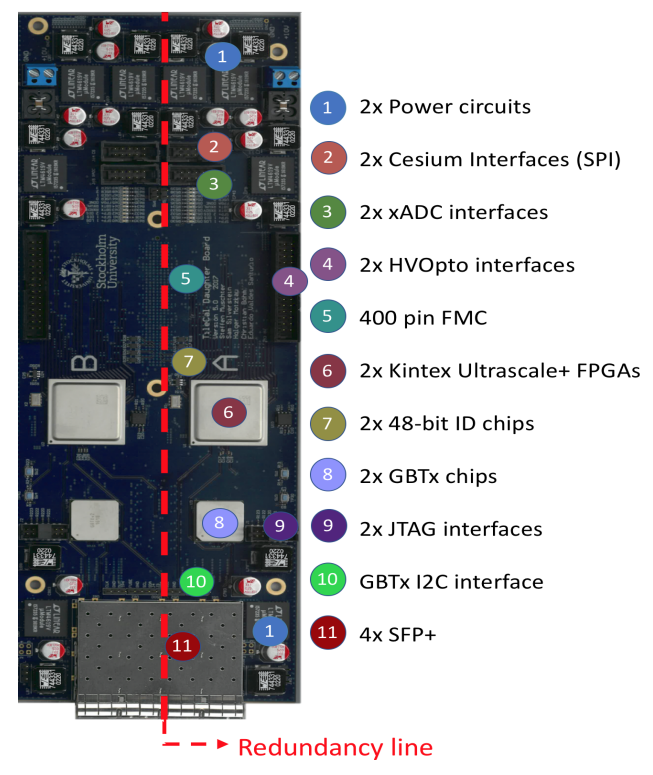

Fig. 3. Daughterboard revision 5.

The DB5 interfaces with the TilePPr by means of four Small Form-factor Pluggable (SFP+) modules servicing four 9.6 Gbps TX lines driven by the GTY transceivers of the two Kintex Ultrascale+ FPGAs, and two 4.8 Gbps RX received by two GBTx chips. Each GBTx receives and recovers eight LHC synchronized clock outputs: two $160 \mathrm{MHz}$ clocks to drive the multi-Gigabit transceivers, two $40 \mathrm{MHz}$ clocks to drive the FPGA's logic and four $40 \mathrm{MHz}$ phase adjusted clocks to drive the ADC and provide timing adjustment. A Triple Modular Redundancy (TMR) protected FPGA firmware formats the digitized two gain PMT data and slow control data in GBTCRC protected words and transmits via the two pairs of $2 \mathrm{x}$ TX links, each running at $9.6 \mathrm{Gbps}$.

\section{TID AND NIEL IRRADIATION TESTS}

Exposure to high levels of radiation are expected due to the placement of the DB in TileCal, therefore the board is required to be certified for handling $20 \mathrm{kRad}$ of the Total Ionizing Dose (TID) and Non-Ionizing Energy Loss (NIEL) of $1.12655 \times 10^{12}-1 \mathrm{MeV}$ equivalent neutron fluence throughout the operation of the HL-LHC. Both of the tests have been

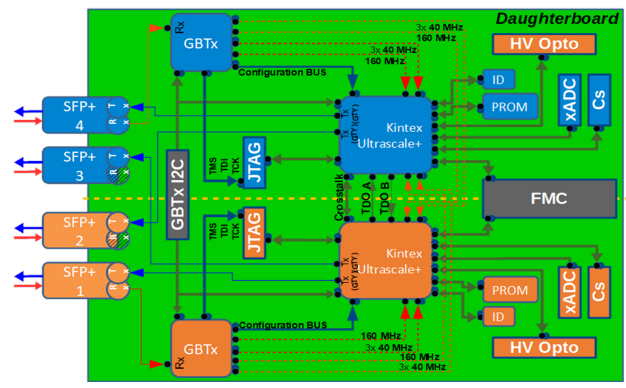

Fig. 4. TileCal DB schematic. [3]

performed [3] according to the ESCC-22900 standard [4] and ATLAS policy on radiation tolerant electronics.

The TID test was performed with a quasi-monochromatic 9 $\mathrm{MeV}$ electron beam in six doses over a time period of one hour for a total dose of $20 \mathrm{kRad}$. The beam with square dimensions $(263 \mathrm{~mm} \times 263 \mathrm{~mm})$ covered the areas of the FPGAs, GBTxs and PROMs. Between each dose, the system was power-cycled and checked for the automatic reconfiguration of the FPGAs from the PROMs. The stability of the monitored voltages and currents for the whole board was sustained throughout the irradiation period, apart from a small drop in the GBTx currents, which had no impact on the functionality of board. No component failure has been observed during the tests. A
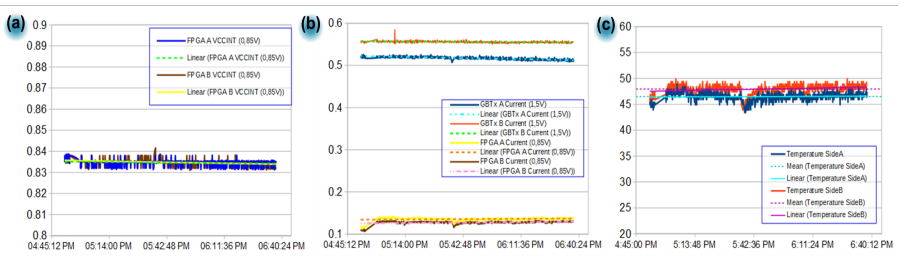

Fig. 5. Tile DB TID test. [3]

quasi-energetic beam of protons with an energy of $52 \mathrm{MeV}$ has been used for the NIEL tests with the aim of a total dose of $9 \times 10^{12} \mathrm{~cm}^{-2}-1 \mathrm{MeV}$ equivalent neutron fluence. The DB and $2 \mathrm{SFP}+$ were covered by a double Gaussian beam profile with $13.5 \mathrm{~cm}$ FWHM distanced $14.8 \mathrm{~cm}$ apart to achieve a homogeneous dose of $10 \%$ along the redundancy line. The board has acquired induced radioactivity during the tests and a cool-down period is required to perform additional studies and fully conclude the test.

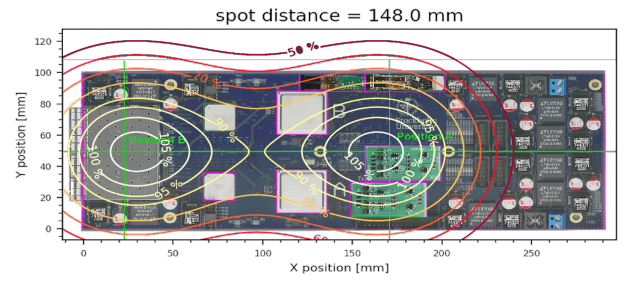

Fig. 6. TileCal DB NIEL test. [3] 


\section{CONCLUSION}

The TID tests has been successful with the stable operation of the board and showing no signs of radiation induced latchup. The results from the NIEL test will be available once the cool-down period is over. Future plans include tests for destructive events by using a $200 \mathrm{MeV}$ proton beam with the size of $9 \times 9 \mathrm{~cm}$ to cover the FPGAs, GBTx and remote JTAG logic. The target flux of $1.1 \times 10^{12} \mathrm{p} / \mathrm{cm}^{2}$ will be achieved by irradiating 2 boards at $5.5 \times 10^{11} \mathrm{p} / \mathrm{cm}^{2}$. Studies for Single Event Effects (SEE) such as Single Event Upsets and Single Event Latch-ups tests will be carried out with $58 \mathrm{MeV}$ and $226 \mathrm{MeV}$ proton beams.

\section{REFERENCES}

[1] ATLAS Collaboration, "Technical Design Report for the Phase-II Upgrade of the ATLAS Tile Calorimeter." https://cds.cern.ch/record/2285583, CERN-LHCC-2017-019, ATLAS-TDR-028, 2018

[2] "GBTX Manual",

https://espace.cern.ch/GBT-Project/GBTX/Manuals/gbtxManual.pdf, 2016

[3] Valdes Santurio, Eduardo and Silverstein, Samuel and Bohm Christian, "ATLAS Tile Calorimeter Link Daughter Board." https://cds.cern.ch/record/2644277, ATL-TILECAL-PROC-2018-014, 2018

[4] "Total dose steady-state irradiation test method ESCC22900", http://escies.org/escc-specs/published/22900.pdf, 2016 